- having two Popes - it was the dendritic cells versus the macrophages." Steinman continued doggedly collecting data, and eventually won over his critics.

Two decades after the discovery of dendritic cells' crucial role, a team led by Hoffmann was investigating why fruitflies, which lack an adaptive immune system, don't succumb to fungal infection. In 1996, they reported that the Toll gene, previously linked to embryo development, was also important for battling infections ${ }^{2}$. Flies with mutations in Toll died when exposed to bacteria or fungi.

At around the same time, a team led by Beutler, then at the University of Texas Southwestern Medical Center in Dallas, had spent six years looking for an immune-system gene in mice that produces a protein to recognize lipopolysaccharide (LPS), a molecule produced by certain bacteria that can cause septic shock. "We were obsessed," says Alexander Poltorak, an immunologist now at Tufts University in Boston, Massachusetts, who worked on the project. "We always thought we would find the gene tomorrow."

The team eventually found its LPSsensing gene, and it looked remarkably like Hoffmann's Toll ${ }^{3}$. Linking the two findings paved the way for the discovery of other Tolllike receptors that sense molecules made by pathogens but not their hosts, and form a critical part of the innate immune system.

The discoveries of dendritic cells and innate immune receptors have already had an impact on medicine. Vaccines are typically administered with an adjuvant, such as a metal, to prompt a rapid immune response. Drug companies such as GlaxoSmithKline are now developing adjuvants that activate Toll-like receptors.

"By doing this we are mimicking what actually happens during an infection without having an infection," says Vincenzo Cerundolo, associate director of the UK Medical Research Council Immunology Unit in Oxford.

Meanwhile, Provenge (made by the biotechnology company Dendreon of Seattle, Washington), the only cellular immune therapy against cancer to be approved by the US Food and Drug Administration, exploits dendritic cells that recognize a molecule produced by prostate tumours. Culturing and reinjecting the cells back into the patient fortifies the immune response against the tumour.

"The reason why the field has progressed so much and is now in the clinic is because we understand how to activate the immune system," says Cerundolo. .

1. Steinman, R. M. \& Cohn, Z. A. J. Exp. Med. 137, 1142-1162 (1973).

2. Lemaitre, B. et al. Cell 86, 973-983 (1996).

3. Poltorak, A. et. al. Science 282, 2085-2088 (1998).
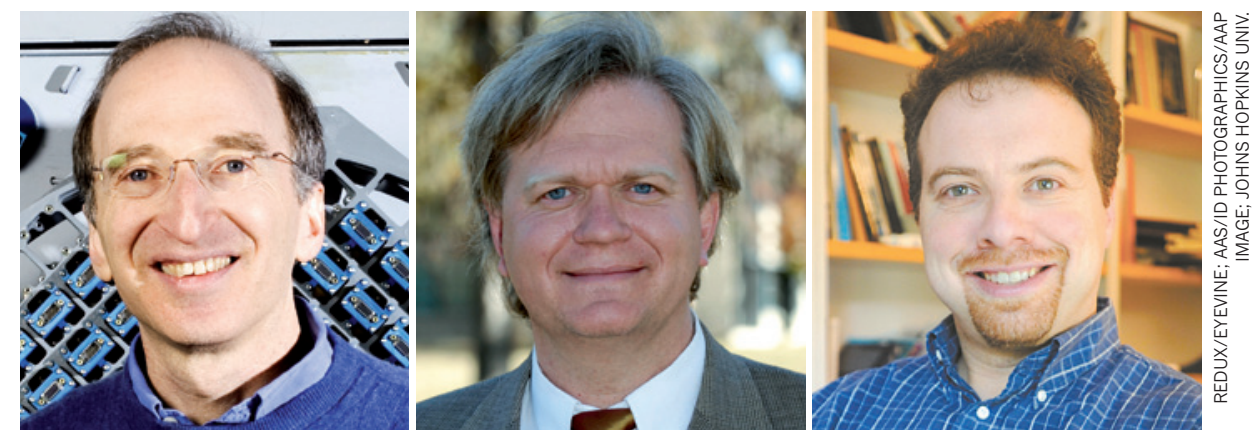

Shining brightly: (from left) Saul Perlmutter, Brian Schmidt and Adam Riess.

NOBEL PRIZE

\title{
Stellar performance nets physics prize
}

Nobel for supernovae signals of accelerating Universe.

\section{BY GEOFF BRUMFIEL}

$\mathrm{T}$ Three astrophysicists have been awarded a Nobel prize for planting a perplexing puzzle at the heart of cosmology.

Half of the Nobel Prize in Physics goes to Saul Perlmutter of Lawrence Berkeley National Laboratory in California for leading a team that discovered that the Universe is expanding at an ever-increasing rate (S. Perlmutter et al. Astrophys. J. 517, 565-586; 1999). Brian Schmidt of the Australian National University in Weston Creek and Adam Riess of the Space Telescope Science Institute in Baltimore, Maryland, share the other half of the prize for independent measurements of the cosmic acceleration (A. G. Riess et al. Astron. J. 116, 1009-1038; 1998), which researchers have struggled to explain ever since.

"I feel kind of weak in the knees," Schmidt told reporters in Sweden via telephone. "It sort of feels like when my children were born."

All three scientists reached their conclusions on the basis of measurements of distant Type Ia supernovae. These occur in very specific types of binary star system, in which a white dwarf star tears matter away from its partner until it gains enough mass to explode. At their peak, Type Ia supernovae always emit roughly the same amount of light, making them useful as 'standard candles' by which to measure vast distances across the cosmos.

In the late 1980s and early 1990s, the prizewinners precisely measured the brightness of these supernovae using newly developed digital sensors. They then compared the brightness to the redshift - the change in colour of the light that results from the motion of the supernovae away from us. Both teams found that the supernovae were dimmer than expected at the measured redshift. The inescapable conclusion was that the Universe was not only expanding - which astronomers first realized in the 1920s - but expanding faster and faster.

Schmidt says that the finding was initially "pretty perplexing". Most astronomers had expected that the Universe's rapid growth following the Big Bang would gradually slow down as gravity pulled distant galaxies towards each other. Yet the discovery was accepted almost immediately by the astronomical community - in part because the idea of a cosmic pressure pushing the Universe outwards had already been mooted by Albert Einstein.

When Einstein applied his general theory of relativity to the Universe as a whole in 1917, his equations included a 'cosmological constant' which described just such an outward force. Over the past decade, observations of the largescale structure of the Universe, together with the cosmic microwave background radiation - the faint afterglow of the Big Bang - have also indicated that the majority of the Universe's energy remains undetected. Today, the astronomical community accepts that about $73 \%$ of the Universe's energy is invested in this cosmic acceleration. Known as dark energy, it remains a largely mysterious force. "Nobody really knows what it is that has been discovered," says Peter Coles, an astrophysicist at Cardiff University, UK.

The predominant view is that dark energy results from quantum fluctuations in the vacuum of space, but efforts to use quantum theory to describe it have so far failed. Other theories, including modifications of gravity, have gained little acceptance.

"It could be none of the above," Coles says. But "we wouldn't be on the trail of this 'none-of-the-above' if it hadn't been for these experiments". 